\title{
Introduction to the Data Analytics Management, Governance, and Compliance Minitrack
}

\author{
Michael Goul \\ W. P. Carey School of Business \\ Arizona State University \\ Michael.Goul@asu.edu
}

\author{
Zhongju Zhang \\ W. P. Carey School of Business \\ Arizona State University \\ Zhongju.Zhang@asu.edu
}

\author{
Jeff Saltz \\ School of Information Studies \\ Syracuse University \\ jsaltz@syr.edu
}

\section{Introduction}

Big Data, data science and analytics have become increasingly important strategic assets because they can help organizations make better decisions, discover new insights, competitively differentiate, and they enable the embedding of intelligence into automated processes so organizations can efficiently respond at the speed of business. They can also provide inter-organizational partnerships with sharable, actionable insights leading to significant innovation.

Organizations need new theories, frameworks and methodologies that can help them:

- $\quad$ Realize strategies and principles for managing Big Data

- Streamline processes to develop and deploy analytical models and machine learning algorithms

- Design new KPIs and deploy actionable dashboards

- Manage and staff data science teams

- Structure and coordinate analytics functions and capabilities within organizations

- Design, staff and provide direction to data and analytics governance committees

- Manage project and deployment risk, and

- Advance analytics capability maturity

Traditional IT project portfolio governance is well established. Effective governance benefits include higher return on assets, investments linked to strategic priorities, less duplication of effort and increased agility. Historical IT portfolio governance helped companies navigate spending and decision-making around IT thereby minimizing investments that were poorly aligned with strategy and too costly given potential benefits. Organizations now face a new set of dilemmas related to advancements in Big Data, data science and analytics governance. With projects popping up throughout organizations' business unit landscapes, there is a new reality that needs to be addressed by vitalizing traditional IT governance approaches that continue to work, and innovating novel solutions when new situations require them.

What is new here? Organizational roles like Chief Data Officer and Chief Analytics Officer are emerging. Funding models for prioritizing analytics opportunities are more frequently being discussed. Centers of Excellence and shared services entities are being created to handle and manage an increasing data science workload. New agile methodologies are emerging, and building an organization-wide culture of evidence-based management is becoming a competitive necessity. Big Data resource investment decisions are becoming more complex with the emergence of the Internet of Things. Effective organizational management and governance of data analytic practices are necessary in order to mitigate risks associated with analytics deployment. Organizations need to capture and manage critical meta-information detailing modeling and environmental assumptions underlying analytics solutions, and they need to establish policies and a culture designed to ensure adherence to the highest ethical standards of data management and predictive model deployment. In addition, organizations need new legal analytics tool familiarity, and there is a need to understand the role of legal analytics in compliance. Unleashing machine learning algorithms that may take on a life of their own may require novel safeguards including ongoing risk mitigation monitoring.

\section{Sessions}

This minitrack examines challenges surrounding theory, frameworks, development, evaluation, and impact of Big Data, data science and analytics 
management, governance, and compliance. The three papers accepted for the minitrack investigate these issues in different ways.

The first paper, titled "Understanding the Changing Role of the Management Accountant in the Age of Industry 4.0 in Germany" by Teuteberg, Bensberg and Buscher, investigates the implications of business processes that undergoing a transformation through digitalization (I4.0) on management accounting by means of a triangulation approach. The results of expert interviews, a literature review and an analysis of job advertisements provide an overview on the development of competencies of Management Accountants (MAs) in Germany. The results highlight a close cooperation between MAs and Data Scientists.

In the second paper titled "Multivariate Unsupervised Machine Learning for Anomaly Detection in Enterprise Applications", by Elsner, Khosroshahi, MacCormack and Lagerström, the authors develop a density-based unsupervised machine learning model to detect anomalies within an enterprise application, based upon data from multiple application performance management systems. The research was conducted in collaboration with a European automotive company, using two months of live application data. They show that their model detects abnormal system behavior more reliably than a commonly used outlier detection technique and provides information for detecting root causes.

In the third paper titled "AI agency risks and their mitigation through business process management: a conceptual framework", Sidorova and Rafiee build on agency theory and identify factors that increase the risk of an agency problem between a principal (a human or an organization) and an AI agent, and then propose a framework for AI agency problem analysis. The framework is illustrated through AI use cases and industry examples. 